\title{
REDUCING THE NUMBER OF FIXED POINTS OF SOME HOMEOMORPHISMS ON NONPRIME 3-MANIFOLDS
}

\author{
XUEZHI ZHAO
}

Received 5 September 2004; Revised 15 March 2005; Accepted 21 July 2005

We will consider the number of fixed points of homeomorphisms composed of finitely many slide homeomorphisms on closed oriented nonprime 3-manifolds. By isotoping such homeomorphisms, we try to reduce their fixed point numbers. The numbers obtained are determined by the intersection information of sliding spheres and sliding paths of the slide homeomorphisms involved.

Copyright (c) 2006 Xuezhi Zhao. This is an open access article distributed under the Creative Commons Attribution License, which permits unrestricted use, distribution, and reproduction in any medium, provided the original work is properly cited.

\section{Introduction}

Nielsen fixed point theory (see $[1,4]$ ) deals with the estimation of the number of fixed points of maps in the homotopy class of any given map $f: X \rightarrow X$. The Nielsen number $N(f)$ provides a lower bound. A classical result in Nielsen fixed point theory is: any map $f: X \rightarrow X$ is homotopic to a map with exactly $N(f)$ fixed points if the compact polyhedron $X$ has no local cut point and is not a 2-manifold. This includes all smooth manifolds with dimension greater than 2 .

It is also an interesting question whether the Nielsen number can be realized as the number of fixed points of a homeomorphism in the isotopy class of a given homeomorphism. In fact, it is just what J. Nielsen expected when he introduced the invariant $N(f)$. Assume that $X$ is a closed manifold. The answer to this question is obviously positive for the unique closed 1-manifold. A positive answer was given by Jiang and Guo [5] for 2-manifolds, and was given by Kelly [7] for manifolds of dimension at least 5.

In [6], Jiang, Wang and Wu proved that for any closed oriented 3-manifold $X$ which is either Haken or geometric, any orientation-preserving homeomorphism $f: X \rightarrow X$ is isotopic to a homeomorphism with $N(f)$ fixed points ([6, Theorem 9.1]). If Thurston's geometric conjecture is true, all nonprime 3-manifolds are of this type.

In this paper, we will consider a certain class of homeomorphisms of closed, oriented 3-manifolds that have a connected sum decomposition into prime factors, namely irreducible manifolds and copies of $S^{2} \times S^{1}$, and at least two factors (nonprime manifolds). 
It is known from work of Kneser and Milnor that in the oriented setting, the prime and irreducible factors of the decomposition are unique. We examine homeomorphisms that can be expressed as the composition of finitely many slide homeomorphisms. A so-called slide homeomorphism is the identity away from a certain stratified open neighborhood, the sliding set, of a torus, and is defined by a family of rotation-like transformations on this set. According to McCullough's result (see [8]), an arbitrary homeomorphism of a reducible 3-manifold can be expressed as the composition of homeomorphisms that comes in four types, one of which is that of slide homeomorphisms.

In [9], the author considered the Nielsen numbers and fixed points of homeomorphisms which are compositions of $m$ slide homeomorphisms on nonprime 3-manifolds. The fixed point index of the complement of the union of the sliding sets was proved to be zero. When $m=2$, we found presentations that are in some sense "standard," for which the fixed point numbers, the fixed point class coordinates and the fixed point indices for all fixed points can be determined. Thus, we were able to give some estimating bounds on the Nielsen numbers of such kinds of homeomorphisms. The present paper is a continuation of [9]. We will generalize the results for $m=2$ there to the case where $m$ can be an arbitrary positive integer. We will focus on a geometrical method to reduce the number of fixed points in any given isotopy class of such a homeomorphism. The lower bound property of Nielsen number implies that our number of fixed points yields an upper bound for Nielsen number.

The remaining sections are organized as follows. In Section 2, we will fix notation which will be used throughout this paper, and recall the definition of slide homeomorphism. In Section 3, we will show (Lemma 3.4) that away from the sliding set, $f$ can be isotoped to a fixed point free homeomorphism by an arbitrary small isotopy. Although each component of this set has zero fixed point index ([9, Theorem 3.2]), the result here is not very obvious because we are considering fixed points up to isotopy rather than homotopy. In Section 4, fixed points over the sliding set are considered. It is argued that $f$ is isotopic to a homeomorphism with finitely many fixed points, and that the size of this fixed point set is expressible in terms self-intersection data for the sliding set (Proposition 4.6). Reducing the number of fixed points for homeomorphisms in the isotopy class of $f$ then involves controlling in some sense the number of self intersections; our main result (Theorem 4.11) gives a lower bound for this number. Finally, a short Section 5 shows that in some cases, one may simplify and "optimize" the sliding set so that the bound in Section 4 can be further lowered, that is, the number of fixed points can be further reduced.

\section{Conventions and notations}

In this section, we will make necessary conventions in notation, which will be used in later sections.

(1) The underlying manifold $M$. In this paper, the manifold $M$ is assumed to be a closed oriented 3-manifold, which is nonprime. It is known that $M$ can be written as a connected sum of finitely many prime 3-manifolds, that is, $M=M_{1} \# M_{2} \# \cdots \# M_{n^{\prime}} \# \cdots \# M_{n^{\prime}+n^{\prime \prime}}$, in which $M_{i}$ is irreducible for $1 \leq i \leq n^{\prime}$ and $M_{i}=S^{2} \times S^{1}$ for $n^{\prime}+1 \leq i \leq n^{\prime}+n^{\prime \prime}$. The nonprime property implies that $n^{\prime}+n^{\prime \prime}>1$. 
Take a 3-sphere and remove $n^{\prime}+2 n^{\prime \prime}$ open discs to obtain a punctured 3-cell $W$ with $n^{\prime}+2 n^{\prime \prime}$ boundary components. We then have that $M=W \cup\left(\cup_{i=1}^{n^{\prime}+n^{\prime \prime}} M_{i}^{\prime}\right)$, where $M_{i}^{\prime}=$ $M_{i}-\operatorname{Int}\left(D_{i}\right)$ for $1 \leq i \leq n^{\prime}$ and $M_{i}^{\prime}=S^{2} \times I$ for $n^{\prime}+1 \leq i \leq n^{\prime}+n^{\prime \prime}$ (see [8]). Each $M_{i}^{\prime}$ admits the orientation coincident with that of $M$, and each $\partial M_{i}^{\prime}$ inherits the orientation of $M_{i}^{\prime}$.

(2) Slide homeomorphisms. Let $S$ be an oriented essential 2-sphere in $M$, which is orientation-preservingly isotopic to a boundary component of $\partial M_{j}^{\prime}$. Let $\alpha: I \rightarrow M$ be a path without self intersection in $M$ such that $\alpha \cap M_{j}^{\prime}=\alpha \cap S=\{\alpha(0), \alpha(1)\}$. Take two regular neighborhoods $N^{\prime}$ and $N^{\prime \prime}\left(N^{\prime} \subset \operatorname{Int}\left(N^{\prime \prime}\right)\right)$ of $\alpha \cup S$ in $M$. Then $\operatorname{Int}\left(N^{\prime \prime}-N^{\prime}\right)$ has two components which are homeomorphic to $S^{2} \times(0,1)$ and $T^{2} \times(0,1)$ respectively. We write the latter as $T(S, \alpha)$.

Pick a coordinate function $c: T(S, \alpha) \rightarrow T^{2} \times(0,1)$, where the points in $T^{2} \times(0,1)$ are labeled by $(\theta, \varphi, t)$, such that the $\theta$-line, $c^{-1}(\theta, *, *)$, is parallel to the oriented path $\alpha$ and the $t$-line $c^{-1}(*, *, t)$ moves radially away from the path $\alpha$ when the value of $t$ is increased.

A slide homeomorphism $s: M \rightarrow M$ determined by $\alpha$ and $S$ is defined by

$$
s(x)= \begin{cases}c^{-1}(\theta+2 \pi t, \varphi, t) & \text { if } x=c^{-1}(\theta, \varphi, t) \in T(S, \alpha), \\ x & \text { otherwise, }\end{cases}
$$

denoted by $s(S, \alpha)$. The sets $T(S, \alpha), S$ and $\alpha$ are said to be respectively the sliding set, sliding sphere and sliding path of $s(S, \alpha)$.

(3) Orientations and isotopies. Since all manifolds under consideration are oriented, including sliding spheres and sliding paths, isotopies here are considered to be ambient and orientation-preserving. For example, if $M=M_{1} \# M_{2}$ is a connected sum of two prime manifolds, $\partial M_{1}^{\prime}$ and $\partial M_{2}^{\prime}$ are not regarded as isotopic.

(4) Fundamental groups and path classes. Consider the construction $M=W \cup\left(\cup_{i=1}^{n^{\prime}+n^{\prime \prime}} M_{i}^{\prime}\right)$ of $M$. We choose a point $x_{0}$ in $W$ as its base point. To any path $\gamma$ with ending points in $W$ there corresponds uniquely an element $\left\langle\gamma_{*} \gamma \gamma_{* *}^{-1}\right\rangle$ in $\pi_{1}\left(M, x_{0}\right)$, where $\gamma_{*}$ and $\gamma_{* *}$ are path from $x_{0}$ to $\gamma(0)$ and $\gamma(1)$ in $W$ respectively. By abuse of notation, we write it simply as $\langle\gamma\rangle$. Choose $x_{j} \in \partial M_{j}^{\prime}$ as base point of $M_{j}^{\prime}$ for $j=1,2, \ldots, n^{\prime}+n^{\prime \prime}$. Thus, each $\pi_{1}\left(M_{j}^{\prime}, x_{j}\right)$ is embedded into $\pi_{1}\left(M, x_{0}\right)$ in a natural way as above, and hence $\pi_{1}\left(M, x_{0}\right)$ is the free product of $\pi_{1}\left(M_{j}^{\prime}, x_{j}\right), j=1,2, \ldots, n^{\prime}+n^{\prime \prime}$. We write simply as $\pi_{1}\left(M, x_{0}\right)=\pi_{1}\left(M_{1}^{\prime}\right) * \pi_{1}\left(M_{2}^{\prime}\right) *$ $\cdots * \pi_{1}\left(M_{n^{\prime}+n^{\prime \prime}}^{\prime}\right)$, which is also equal to $\pi_{1}\left(M_{1}\right) * \pi_{1}\left(M_{2}\right) * \cdots * \pi_{1}\left(M_{n^{\prime}+n^{\prime \prime}}\right)$.

(5) The homeomorphism $f$. From now on, $f$ is assumed to be a homeomorphism composed of finitely many slide homeomorphisms, that is, $f=s\left(S_{m}, \alpha_{m}\right) \circ s\left(S_{m-1}, \alpha_{m-1}\right) \circ$ $\cdots \circ s\left(S_{1}, \alpha_{1}\right)$. The union $\cup_{j=1}^{m} T\left(S_{j}, \alpha_{j}\right)$ of all sliding sets is said to the sliding set of $f$. For a simplification in notation, we write $s_{m^{\prime \prime} \cdots m^{\prime}}$ for the composition $s\left(S_{m^{\prime \prime}}, \alpha_{m^{\prime \prime}}\right) \circ s\left(S_{m^{\prime \prime}-1}\right.$, $\left.\alpha_{m^{\prime \prime}-1}\right) \circ \cdots \circ s\left(S_{m^{\prime}}, \alpha_{m^{\prime}}\right)$ for any $m^{\prime}$ and $m^{\prime \prime}\left(1 \leq m^{\prime}<m^{\prime \prime} \leq m\right)$. In particular, $s\left(S_{j}, \alpha_{j}\right)$ is simply written as $s_{j}$.

(6) General position. Consider the slide homeomorphisms whose composition is $f$. We can ensure the sliding paths $\alpha_{1}, \alpha_{2}, \ldots, \alpha_{m}$, and sliding spheres $S_{1}, S_{2}, \ldots, S_{m}$ are in general position relative to the set $\cup_{j=1}^{m}\left\{\alpha_{j}(0), \alpha_{j}(1)\right\}$. Thus, these sliding paths have no intersection, and $\alpha_{i}$ intersects with $S_{j}$ transversally for $i \neq j$. Since each sliding sphere is isotopic to a component of $-\partial W$, we can arrange these sliding spheres to be disjoint. In this situation, if each sliding set $T\left(S_{j}, \alpha_{j}\right)$ is in a small neighborhood of $\alpha_{j} \cup S_{j}$, the number 


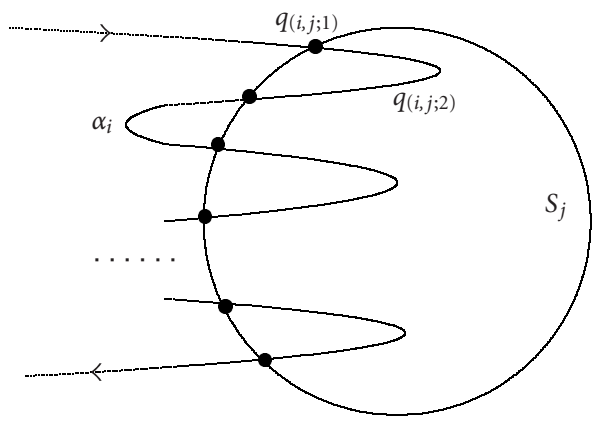

Figure 2.1

of components of intersection of two sliding sets $T\left(S_{j^{\prime}}, \alpha_{j^{\prime}}\right)$ and $T\left(S_{j^{\prime \prime}}, \alpha_{j^{\prime \prime}}\right)$ is equal to the number of points in $\left(\alpha_{j^{\prime}} \cup S_{j^{\prime}}\right) \cap\left(\alpha_{j^{\prime \prime}} \cup S_{j^{\prime \prime}}\right)$ for all $j^{\prime}$ and $j^{\prime \prime}$ with $j^{\prime} \neq j^{\prime \prime}$. In this situation, we say that the sliding set $\cup_{j=1}^{m} T\left(S_{j}, \alpha_{j}\right)$ of $f$ is in general position.

(7) Components $B_{(*, * ; *)}$ of the intersection of sliding sets. If the sliding set $\cup_{j=1}^{m} T\left(S_{j}, \alpha_{j}\right)$ is in general position, the points in $\alpha_{i} \cap S_{j}(i \neq j)$ are denoted by $q_{(i, j ; 1)}, q_{(i, j ; 2)}, \ldots$, $q_{\left(i, j ;\left|\alpha_{i} \cap S_{j}\right|\right)}$ (see Figure 2.1), where the last subscript indicates the order in $\alpha_{i} \cap S_{j}$ along the direction of $\alpha_{i}$, that is, $\alpha_{i}^{-1}\left(q_{\left(i, j ; k^{\prime}\right)}\right)<\alpha_{i}^{-1}\left(q_{\left(i, j ; k^{\prime \prime}\right)}\right)$ in $I=[0,1]$ if and only if $k^{\prime}<$ $k^{\prime \prime}$. The corresponding components of $T\left(S_{i}, \alpha_{i}\right) \cap T\left(S_{j}, \alpha_{j}\right)$ nearby are written as $B_{(i, j ; 1)}$, $B_{(i, j ; 2)}, \ldots, B_{\left(i, j ;\left|\alpha_{i} \cap S_{j}\right|\right)}$. Obviously, we have

Proposition 2.1. If the sliding set $\cup_{j=1}^{m} T\left(S_{j}, \alpha_{j}\right)$ is in general position, then each $B_{(*, * ; *)}$ is homeomorphic to a solid torus, and $T\left(S_{i}, \alpha_{i}\right) \cap T\left(S_{j}, \alpha_{j}\right)=\left(\sqcup_{k=1}^{\left|\alpha_{i} \cap S_{j}\right|} B_{(i, j ; k)}\right) \sqcup\left(\sqcup_{l=1}^{\left|\alpha_{j} \cap S_{i}\right|} B_{(j, i ; l)}\right)$ for any $i$ and $j$, where $i, j=1,2, \ldots, m$ with $i \neq j$.

\section{Removing fixed points on the complement of sliding set}

Consider our homeomorphism $f$. Since the fixed point set of each slide homeomorphsim $s_{i}$ is just $M-T\left(S_{i}, \alpha_{i}\right)$, the points in the complement $M-\cup_{j=1}^{m} T\left(S_{j}, \alpha_{j}\right)$ of the sliding set of $f$ are totally contained in the fixed point set of $f$. In [9], we proved that this isolated fixed point set has zero fixed point index. In this section, we will show that this fixed point set can be removed by arbitrary small isotopy.

The following definition is originally from [2].

Definition 3.1. Let $\Gamma: N \rightarrow T N$ be a vector field on a compact smooth $n$-manifold $N$. The manifold $N$ is said to be a manifold with corners for the vector field $\Gamma$ if $\Gamma$ has no singular points on $\partial N$ and if $\partial N$ can be considered a union of $(n-1)$-manifolds (with boundaries) $\partial_{o} N, \partial_{+} N$ and $\partial_{-} N$ with $\partial_{+} N \cap \partial_{-} N=\varnothing$ such that $\Gamma(x)$ is tangent to $\partial_{o} N$ for $x \in \partial_{o} N$, points inward to $N$ for $x \in \partial_{-} N$ and points outward from $N$ for $x \in \partial_{+} N$.

Clearly, for an $n$-manifold $N$ with corners, both of $\partial_{+} N \cap \partial_{o} N$ and $\partial_{-} N \cap \partial_{o} N$ are $(n-2)$-dimensional closed manifolds. A simple example is the following. 
Example 3.2. A constant vector field on $R^{2}$ is given by $\Gamma(x, y)=(1,0)$. Then the subset $N=[0,1] \times[0,1]$ is a manifold with corners for such a vector field $\Gamma$, with $\partial_{0} N=[0,1] \times$ $\{0,1\}, \partial_{-} N=\{0\} \times[0,1]$ and $\partial_{+} N=\{1\} \times[0,1]$.

The next lemma is a kind of generalization of the Poincaré-Hopf vector field index theorem. There are some similar statements in dynamical system theory, see for example [3, Lemma A.1.3].

LEMMA 3.3. Let $N$ be a 3-manifold with corners for a vector field $\Gamma$. If the boundary $\partial N$ is a disjoint union of $m$-copies of a sphere such that $\partial_{o} N$ is a disjoint union of $m$-copies of an annulus, and either $\partial_{+} N$ or $\partial_{-} N$ is a disjoint union of $m$-copies of a disc, then we can change $\Gamma$ relative to a neighborhood of $\partial N$ in $N$ into a nonsingular vector field $\Gamma^{\prime}$.

Proof. Through a coordinate function, each component of $\partial N$ can be regarded as one of the following:

$$
C_{k}=\left\{(x, y, z):|x| \leq 4,(y-8 k)^{2}+z^{2}=4 \text { or } x= \pm 4,(y-8 k)^{2}+z^{2} \leq 4\right\},
$$

where $k=1,2, \ldots, m$. Since $\partial_{+} N \cap \partial_{-} N=\varnothing$, we may assume that

$$
\begin{aligned}
& \partial_{o} N=\cup_{k=1}^{m}\left\{(x, y, z):|x| \leq 4,(y-8 k)^{2}+z^{2}=4\right\}, \\
& \partial_{-} N=\cup_{k=1}^{m}\left\{(x, y, z): x=4,(y-8 k)^{2}+z^{2} \leq 4\right\}, \\
& \partial_{+} N=\cup_{k=1}^{m}\left\{(x, y, z): x=-4,(y-8 k)^{2}+z^{2} \leq 4\right\} .
\end{aligned}
$$

Regard a neighborhood of $\partial N$ as a subset outside of the cylinders:

$$
D_{k}=\left\{(x, y, z):|x| \leq 4,(y-8 k)^{2}+z^{2} \leq 4\right\}, \quad k=1,2, \ldots, m .
$$

Since $N$ is a manifold with corners for the vector field $\Gamma, \Gamma$ points inward for the cylinders (outward for $N$ ) at $\partial_{+} N$ and points outward for the cylinders (inward for $N$ ) at $\partial_{-} N$. We have that $\Gamma(p) \in\{(x, y, z): x>0\}$ for $p \in \partial_{+} N \cup \partial_{-} N$. It is not difficult to prove that the restriction of $\Gamma$ at each component of $\partial N$, as a map from a sphere to $R^{3}-\{0\}$, has zero degree. Hence, we can extend $\Gamma$ to the union $\cup_{k=1}^{m} D_{k}$ such that there is no singular point on $\cup_{k=1}^{m} D_{k}$.

Since $N \cup\left(\cup_{k=1}^{m} D_{k}\right)$ is a closed 3-manifold, its Euler characteristic number is zero. Using standard methods in differential topology, we can deform $\Gamma$ into a nonsingular $\Gamma^{\prime}$ relative to a neighborhood of $\cup_{k=1}^{m} D_{k}$. Then $\left.\Gamma^{\prime}\right|_{N}$ is our desired vector field.

Using this lemma, we can prove the following lemma. 
Lemma 3.4. Assume that the sliding set of $f$ is in general position. Given any positive number $\varepsilon$, there is an isotopy $F: M \times I \rightarrow M$ from $f$ to $f^{\prime}$ satisfying:

(i) $d(F(x, t), f(x))<\varepsilon$ for any $x \in M$ and any $t \in I$,

(ii) the support set $\{x \in M: F(x, t) \neq f(x)$ for some $t \in I\}$ of $F$ is contained in the $\varepsilon$ neighborhood $N_{\varepsilon}\left(M-\cup_{j=1}^{m} T\left(S_{j}, \alpha_{j}\right)\right)$ of the complement of the sliding set $\cup_{j=1}^{m} T\left(S_{j}, \alpha_{j}\right)$ in $M$,

(iii) $\operatorname{Fix}\left(f^{\prime}\right)=\operatorname{Fix}(f)-\left(M-\cup_{j=1}^{m} T\left(S_{j}, \alpha_{j}\right)\right)$.

Proof. Clearly, we can regard a neighborhood $N(\partial W)$ of $\partial W$ in $M$ as a subset of $R^{3}$ so that $\partial W=-\cup_{j=1}^{n^{\prime}+2 n^{\prime \prime}} C_{j}$, where

$$
\begin{gathered}
C_{j}=\left\{(x, y, z) \in R^{3}:(x, y, z):|x| \leq 4,(y-8 j)^{2}+z^{2}=4\right. \\
\text { or } \left.x= \pm 4,(y-8 j)^{2}+z^{2} \leq 4\right\},
\end{gathered}
$$

having the orientation induced from $R^{3}$. Since $\partial W=-\cup_{j=1}^{n^{\prime}+n^{\prime \prime}} \partial M_{j}^{\prime}$, we may arrange so that $\partial M_{j}^{\prime}=C_{j}$ for $1 \leq j \leq n^{\prime} ; \partial M_{n^{\prime}+j}^{\prime}=C_{n^{\prime}+2 j-1} \cup C_{n^{\prime}+2 j}$. The set $W$ is located outside of these $C_{j}$ 's with respect to the given orientation of $C_{j}$ 's.

Clearly, we can construct a vector field $\Gamma_{0}: M \rightarrow T M$ on $M$ so that $\Gamma_{0}(p)=\{1,0,0\}$ for any $p$ in the neighborhood $N(\partial W)$ of $\partial W$ in $M$, where

$$
\begin{gathered}
N(\partial W)=\cup_{k=1}^{n^{\prime}+2 n^{\prime \prime}}\left\{(x, y, z) \in R^{3}:(x, y, z):|x| \leq 3,1 \leq(y-8 k)^{2}+z^{2} \leq 9\right. \\
\text { or } \left.3 \leq|x| \leq 5,(y-8 k)^{2}+z^{2} \leq 9\right\} .
\end{gathered}
$$

Thus, $W$ and all $M_{j}^{\prime}$ s are manifolds with corners for $\Gamma_{0}$. Apply Lemma 3.3 to $W$ and all $M_{j}^{\prime}$ 's, we will get a nonsingular vector field $\Gamma: M \rightarrow T M$ on $M$ so that $\Gamma(p)=\{1,0,0\}$ for any $p \in N(\partial W)$.

By definition of slide homeomorphism, each sliding sphere $S_{k}$ is isotopic to a $C_{j}$ in $M$. We then have a well-defined correspondence $\mu:\{1,2, \ldots, m\} \rightarrow\left\{1,2, \ldots, n^{\prime}+2 n^{\prime \prime}\right\}$ such that $S_{k}$ is isotopic to $C_{\mu(k)}$ in $M$ for any $k=1,2, \ldots, m$.

We take $S_{k}$ to be the sphere outside of $C_{\mu(k)}$ by a distance of $\nu_{k}\left(0<v_{k}<1\right)$. Moreover, we can arrange these $\nu_{1}, \nu_{2}, \ldots, v_{m}$ to have distinct values. Each sliding path $\alpha_{k}$ attaches the corresponding sliding sphere $S_{k}$ at "top" and "bottom" perpendicularly. More precisely, $\alpha_{k}(u)=\left(\nu_{k}, 8 \mu(k), 2+\nu_{k}+u\right)$ and $\alpha_{k}(1-u)=\left(\nu_{k}, 8 \mu(k),-2-\nu_{k}+1-u\right)$ for small $u \in$ I. Each point $q_{(j, k ; *)}$ in $\alpha_{j} \cap S_{k}$ lies on $\left(x\left(q_{(j, k ; *)}\right), 8 \mu(k)+2+v_{k}, 0\right)$, where all possible $x\left(q_{(j, k ; *)}\right)$ are distinct numbers in $(-1,1)$ (see Figure 3.1).

We can make such an arrangement because any two sliding spheres and any two sliding paths have no intersection by the general position assumption. We then arrange the sliding set to lie in a sufficiently small neighborhood of $\cup_{j=1}^{m}\left(\alpha_{j} \cup S_{j}\right)$.

Let $\xi: M \times R \rightarrow M$ be the flow generated by $\Gamma$. We will show that $\xi(f(p), t) \neq p$ for all points $p$ in a small $\eta$-neighborhood $N_{\eta}$ of $M-\cup_{j=1}^{m} T\left(S_{j}, \alpha_{j}\right)$ in $M$ provided $t$ is small enough.

Case 1. If $p \in M-\cup_{i=1}^{m} T\left(S_{i}, \alpha_{i}\right)$, then $f(p)=p$. Since $\Gamma$ has no zero, we have that $\xi(f(p)$, $t)=\xi(p, t) \neq p$ when $t$ is small enough. 


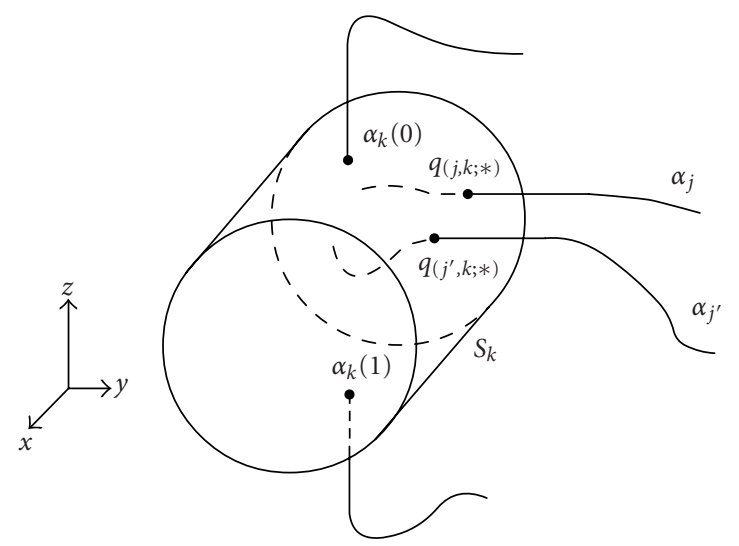

Figure 3.1

Case 2. If $p \in \cup_{i=1}^{m} T\left(S_{i}, \alpha_{i}\right)$, then there is a unique smallest number $j$ with $p \in T\left(S_{j}, \alpha_{j}\right)$. There are two subcases.

Subcase 2.1. If $s_{j}(p) \notin \cup_{i=j+1}^{m} T\left(S_{i}, \alpha_{i}\right)$, then $f(p)=s_{j}(p)$. By general position, we can arrange $\alpha_{j}$ so that $\Gamma\left(\alpha_{j}(u)\right)$ does not parallel to the tangent vector of $\alpha_{j}(u)$ at $u$ for all $u \in I$. Thus, $\xi(\cdot, t)$ will not push along (or opposite) to the direction that $s_{j}$ does. It follows that $\xi(f(p), t) \neq p$ when $p$ is closed to the boundary $\partial T\left(S_{j}, \alpha_{j}\right)$ of $T\left(S_{j}, \alpha_{j}\right)$ (see Figure 3.2).

Subcase 2.2. If $s_{j}(p) \in \cup_{i=j+1}^{m} T\left(S_{i}, \alpha_{i}\right)$, then there is a unique smallest number $k$ with $k>j$ such that $s_{j}(p) \in T\left(S_{k}, \alpha_{k}\right)$. Notice that $p$ is close to $\partial\left(\cup_{i=1}^{m} T\left(S_{i}, \alpha_{i}\right)\right)$. We have that $s_{j}(p)$ is also close to $\partial T\left(S_{k}, \alpha_{k}\right)$ because the difference between $p$ and $s_{j}(p)$ is small, so $s_{k} \circ s_{j}(p)$ will not meet any sliding set other than $T\left(S_{k}, \alpha_{k}\right)$ and $T\left(S_{j}, \alpha_{j}\right)$. It follows that $f(p)=s_{k} \circ s_{j}(p)$.

The component of $T\left(S_{k}, \alpha_{k}\right) \cap T\left(S_{j}, \alpha_{j}\right)$ around $p$ and $f(p)$ have two types: $B_{(k, j ; *)}$ and $B_{(j, k ; *)}$. In the first type, we explain the behavior of $\xi(f(p), t)$ in two parts of Figure 3.3. The first two stages from $p$ to $s_{k} \circ s_{j}(p)$ is shown on the left part. The last stage is illustrated in the right part, where $s_{j}(p)$ is behind $f(p)=s_{k} \circ s_{j}(p)$. Let $p=\left(x_{p}, y_{p}, z_{p}\right)$, we have

$$
\left(x_{p}, y_{p}, z_{p}\right) \stackrel{s_{j}}{\longmapsto}\left(x_{p}, y_{p}^{\prime}, z_{p}^{\prime}\right) \stackrel{s_{k}}{\longrightarrow}\left(x_{p}, y_{p}^{\prime \prime}, z_{p}^{\prime}\right) \stackrel{\xi(\cdot, t)}{\longrightarrow}\left(x_{p}^{\prime \prime \prime}, y_{p}^{\prime \prime}, z_{p}^{\prime}\right) .
$$

This implies that in $R^{3}, p$ and $\xi(f(p), t)$ will have different $x$-values when $t$ is small enough. It follows that $\xi(f(p), t) \neq p$. The proof for the type $B_{(j, k ; *)}$ is the same.

Define an isotopy $F_{\delta, \eta}: M \times I \rightarrow M$ by

$$
F_{\delta, \eta}(p, t)= \begin{cases}\xi(p, \delta t) & \text { if } p \in M-\cup_{j=1}^{m} T\left(S_{j}, \alpha_{j}\right), \\ \xi\left(p, \max \left\{\eta-d\left(p, \cup_{j=1}^{m} \partial T\left(S_{j}, \alpha_{j}\right)\right), 0\right\} \delta t\right) & \text { if } p \in \cup_{j=1}^{m} T\left(S_{j}, \alpha_{j}\right) .\end{cases}
$$




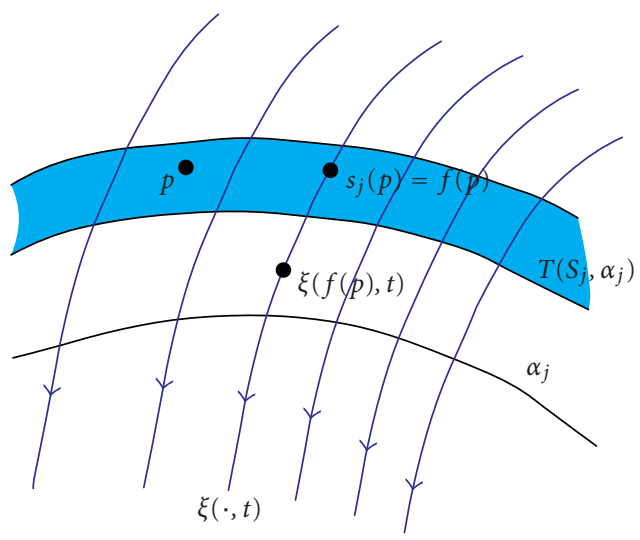

Figure 3.2
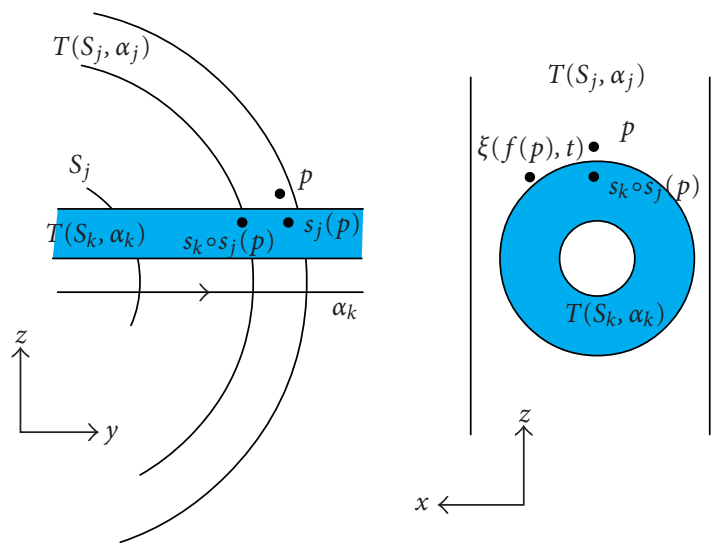

Figure 3.3

Note that the arguments for $\xi$ still work for $F_{\delta, \eta}$, so we can prove that $F_{\delta, \eta}(f(p), t) \neq p$ for all $t \in I$ and $p$ in the $\eta$-neighborhood $N_{\eta}$ of $M-\cup_{j=1}^{m} T\left(S_{j}, \alpha_{j}\right)$ in $M$. Thus, when $\delta$ and $\eta$ are small enough, $F_{\delta, \eta}$ will be a desired isotopy.

Corollary 3.5. Any slide homeomorphism is isotopic to a fixed point free map.

\section{Fixed points on sliding sets}

In this section, we try to reduce the fixed points of the homeomorphism $f$ on its sliding set $\cup_{j=1}^{m} T\left(S_{j}, \alpha_{j}\right)$. For an arbitrary fixed point $x$ of $f$ on its sliding set, we examine its "trace" $x, s_{1}(x), s_{21}(x), \ldots, s_{m \cdots 1}(x)$ under the sliding homeomorphisms composing $f$. Lemma 4.1 will show that the sliding sets of individual slide homeomorphism meeting this trace is totally determined by $x$ itself provided that each sliding set $T\left(S_{j}, \alpha_{j}\right)$ is small enough. Hence, a fixed point $x$ will determine a unique sequence consisting of the 
components of the intersection of sliding sets, which we call the accompanying sequence (Proposition 4.2). All the possible accompanying sequence will be given in Lemma 4.3. Next, we will isotope the given homeomorphism $f$ so that different fixed points on sliding set of $f$ have different accompanying sequences (Lemma 4.4). When the sliding set of $f$ is in general position, there is a unique point $\left(\alpha_{i} \cup S_{i}\right) \cap\left(\alpha_{j} \cup S_{j}\right)$ near an arbitrary component of $T\left(S_{i}, \alpha_{i}\right) \cap T\left(S_{j}, \alpha_{j}\right)$. Thus, in some sense, reducing the number of fixed points is equivalent to reducing the number of intersection points between the sliding paths and sliding spheres. The minimal number $\operatorname{MI}\left(\left\{\alpha_{1}, \ldots, \alpha_{m}\right\},\left\{S_{1}, \ldots, S_{m}\right\}\right)$ of the intersection of sliding paths and sliding spheres gives a possible number of fixed points for homeomorphisms in the isotopy class of $f$ (Theorem 4.11). Since the Nielsen number $N(f)$ is a lower bound of the number of fixed points for maps in the homotopy class of $f$, the minimal number $M I\left(\left\{\alpha_{1}, \ldots, \alpha_{m}\right\},\left\{S_{1}, \ldots, S_{m}\right\}\right)$ also provides an upper bound of $N(f)$.

Lemma 4.1. If any three of these sliding sets $T\left(S_{j}, \alpha_{j}\right)$ 's have no common points, then to each fixed point $x$ of $f$ there is associated a unique sub-sequence $\left\{i_{1}, i_{2}, \ldots, i_{k}\right\}$ of $\{1,2, \ldots, m\}$ with $k \geq 2$ such that $s_{i_{k}} \circ \cdots \circ s_{i_{2}} \circ s_{i_{1}}(x)=x \in T\left(S_{i_{1}}, \alpha_{i_{1}}\right)$, and such that $s_{i_{j-1}} \circ \cdots \circ s_{i_{2}} \circ s_{i_{1}}(x) \in$ $T\left(S_{i_{j}}, \alpha_{i_{j}}\right)$ for $j=2,3, \ldots, k$.

Proof. Let $x$ be a fixed point of $f$ in $\cup_{i=1}^{m} T\left(S_{i}, \alpha_{i}\right)$. There is a unique minimal $i$ such that $x \in T\left(S_{i}, \alpha_{i}\right)$. We write this number as $i_{1}$. A sequence $\left\{i_{1}, i_{2}, \ldots, i_{k}\right\}$ will be defined inductively:

$$
i_{j}=\min \left\{n: n>i_{j-1}, s_{i_{j-1}} \circ \cdots \circ s_{i_{1}}(x) \in T\left(S_{j}, \alpha_{j}\right)\right\}
$$

Since $x \in T\left(S_{i_{1}}, \alpha_{i_{1}}\right)$, we have $s_{i_{1}}(x) \neq x$. If there was no such a number $i_{2}, s_{i_{1}}(x) \notin T\left(S_{i}, \alpha_{i}\right)$ for all $i>i_{1}$. Thus, $f(x)=s_{m \cdots 1}(x)=s_{m \cdots i_{1}}(x)=s_{i_{1}}(x)$. This would contradict the fact that $x$ is a fixed point of $f$, so we always have that $k \geq 2$.

By definition of $i_{j}$, we have that $s_{n \cdots 1}(x)=s_{i_{1}}(x)$ if $i_{1} \leq n<i_{2}$, and that $s_{n \cdots 1}(x)=$ $s_{i_{2}} \circ s_{i_{1}}(x)$ if $i_{2} \leq n<i_{3}$. Inductively, we will get that $s_{n \cdots 1}(x)=s_{i_{p-1}} \circ \cdots \circ s_{i_{2}} \circ s_{i_{1}}(x)$ if $i_{p-1} \leq n<i_{p}$.

When our induction stops at a stage $i_{p}$, we have that $s_{i_{p} \cdots 1}(x)=s_{i_{p}} \circ \cdots \circ s_{i_{2}} \circ s_{i_{1}}(x)$ does not lie in any sliding set $T\left(S_{n}, \alpha_{n}\right)$ with $n>i_{p}$, so $s_{m \cdots i_{p}} \circ s_{i_{p-1}} \circ \cdots \circ s_{i_{1}}(x)=s_{i_{p}} \circ$ $s_{i_{p-1}} \circ \cdots \circ s_{i_{1}}(x)$. It follows that $f(x)=s_{m \cdots 1}(x)=s_{i_{p}} \circ s_{i_{p-1}} \circ \cdots \circ s_{i_{1}}(x)$. This point is just $x$ because $x$ is a fixed point of $f$. Thus, this $i_{p}$ is the final number, say $i_{k}$, in our subsequence of $\{1,2, \ldots, m\}$.

Let us prove the uniqueness of such a subsequence. If there is another subsequence $\left\{j_{1}, j_{2}, \ldots, j_{l}\right\}$ satisfying the same conditions as $\left\{i_{1}, i_{2}, \ldots, i_{k}\right\}$, then we will get that $x \in$ $T\left(S_{j_{1}}, \alpha_{j_{1}}\right)$. Since any three of the sliding sets have no common points, $j_{1}$ is equal to either $i_{1}$ or $i_{k}$. If the last case happens, that is, $j_{1}=i_{k}$, by the choice of $i_{k}$, we have that $s_{j}(x)=x$ for all $j>i_{k}$. Thus, there would be no $j_{2}$. It follows that $i_{1}=j_{1}$.

Assume that $j_{p}=i_{p}$ for $p=1,2, \ldots, n-1$. By the property of the subsequence $\left\{i_{1}, i_{2}, \ldots\right.$, $\left.i_{k}\right\}$, we have $s_{i_{n-1}} \circ \ldots \circ s_{i_{1}}(x) \in T\left(S_{i_{n}}, \alpha_{i_{n}}\right)$; by the property of the subsequence $\left\{j_{1}, j_{2}, \ldots, j_{l}\right\}$, we have $s_{j_{n-1}} \circ \ldots \circ s_{j_{1}}(x) \in T\left(S_{j_{n}}, \alpha_{j_{n}}\right)$. Our assumption implies that $s_{i_{n-1}} \circ \ldots \circ s_{i_{1}}(x)$ and $s_{j_{n-1}} \circ \ldots \circ s_{j_{1}}(x)$ are the same point. Since this point lies in the image of $T\left(S_{i_{n-1}}, \alpha_{i_{n-1}}\right)=$ $T\left(S_{j_{n-1}}, \alpha_{j_{n-1}}\right)$ under homeomorphism $s_{i_{n-1}}=s_{j_{n-1}}$. It also lies in $T\left(S_{i_{n-1}}, \alpha_{i_{n-1}}\right)$. Since any 
three of the sliding sets have no common points, $T\left(S_{i_{n}}, \alpha_{i_{n}}\right), T\left(S_{j_{n}}, \alpha_{j_{n}}\right)$ and $T\left(S_{i_{n-1}}, \alpha_{i_{n-1}}\right)$ are at most two different sets. Because $i_{n} \neq i_{n-1}$ and $j_{n} \neq j_{n-1}=i_{n-1}$, the unique possibility is that $j_{n}=i_{n}$. Thus, we can prove by induction that $j_{n}=i_{n}$ for $n=1,2, \ldots, \min \{k, l\}$.

It remains to show that $k=l$. If $k<l$, then from the property of the subsequence $\left\{j_{1}, j_{2}, \ldots, j_{l}\right\}$, we have that $s_{j_{k}} \circ \cdots \circ s_{j_{1}}(x) \in T\left(S_{j_{k+1}}, \alpha_{j_{k+1}}\right)$. Since we have proved that $j_{n}=i_{n}$ for $n=1,2, \ldots, k, s_{j_{k}} \circ \cdots \circ s_{j_{1}}(x)=s_{i_{k}} \circ \cdots \circ s_{i_{1}}(x)=x$. Thus, $x$ lies in $T\left(S_{i_{1}}, \alpha_{i_{1}}\right) \cap$ $T\left(S_{i_{k}}, \alpha_{i_{k}}\right) \cap T\left(S_{j_{k+1}}, \alpha_{j_{k+1}}\right)$. Since $i_{k}>i_{1}, j_{k+1}$ is equal to either $i_{1}=j_{1}$ or $i_{k}=j_{k}$. This is a contradiction. Symmetrically, the case $k>l$ cannot happen.

For such a fixed point $x$, we write $B_{1}$ for the component of $T\left(S_{i_{k}}, \alpha_{i_{k}}\right) \cap T\left(S_{i_{1}}, \alpha_{i_{1}}\right)$ containing $x$, and write $B_{j}, j=2,3, \ldots, k$, for the component of $T\left(S_{i_{j-1}}, \alpha_{i_{j-1}}\right) \cap T\left(S_{i_{j}}, \alpha_{i_{j}}\right)$ containing $s_{i_{j-1}} \circ \cdots \circ s_{i_{2}} \circ s_{i_{1}}(x)$. The sequence $\left\{B_{1}, B_{2}, \ldots, B_{k}\right\}$ is said to be the accompanying sequence of $x$ in the components of the intersection of sliding sets. Clearly, the set $\left\{B_{1}, B_{2}, \ldots, B_{k}\right\}$ itself is just the set of all components of the intersection of sliding sets containing $s_{n \cdots 1}(x)$ for some $n$. In other words, we have

Proposition 4.2. Let $x$ be a fixed point of $f$ and $\left\{i_{1}, i_{2}, \ldots, i_{k}\right\}$ be its associated sub-sequence of $\{1,2, \ldots, m\}$. Let $\left\{B_{1}, B_{2}, \ldots, B_{k}\right\}$ be a set consisting of some components of the intersection of sliding sets such that $B_{1}$ is a component of $T\left(S_{i_{k}}, \alpha_{i_{k}}\right) \cap T\left(S_{i_{1}}, \alpha_{i_{1}}\right)$, and such that $B_{j}, j=$ $2,3, \ldots, k$, is a component of $T\left(S_{i_{j-1}}, \alpha_{i_{j-1}}\right) \cap T\left(S_{i_{j}}, \alpha_{i_{j}}\right)$. Assume that any three of these sliding sets have no common points. Then, $\left\{B_{1}, B_{2}, \ldots, B_{k}\right\}$ is the accompanying sequence of the fixed point $x$ of $f$ if and only if $x$ belongs to the following set:

$$
s_{i_{k}} \circ s_{i_{k-1}} \circ \cdots \circ s_{i_{1}}\left(B_{1}\right) \cap s_{i_{k}} \circ s_{i_{k-1}} \circ \cdots \circ s_{i_{2}}\left(B_{2}\right) \cap \cdots \cap s_{i_{k}}\left(B_{k}\right) \cap B_{1} .
$$

Lemma 4.3. Assume that the sliding set $\cup_{j=1}^{m} T\left(S_{j}, \alpha_{j}\right)$ of $f$ is in general position. If $s_{j}\left(B_{(i, j ; k)}\right)$ $\cap B_{\left(i^{\prime}, j ; k^{\prime}\right)}=\varnothing$ unless $i=i^{\prime}$ and $k=k^{\prime}$, then the accompanying sequence of each fixed point of $f$ in sliding sets has either one of the following forms:

$$
\begin{aligned}
& \left\{B_{\left(i_{k}, i_{1} ; *\right)}, B_{\left(i_{1}, i_{2} ; *\right)}, B_{\left(i_{2}, i_{3} ; *\right)}, \ldots, B_{\left(i_{k-1}, i_{k} ; *\right)}\right\}, \\
& \left\{B_{\left(i_{1}, i_{k} ; *\right)}, B_{\left(i_{2}, i_{1} ; *\right)}, B_{\left(i_{3}, i_{2} ; *\right)}, \ldots, B_{\left(i_{k}, i_{k-1} ; *\right)}\right\},
\end{aligned}
$$

where $1 \leq i_{1}<i_{2}<\cdots<i_{k} \leq m$ (see Figure 4.1).

Proof. Let $x$ be a fixed point of $f$ in the sliding set with accompanying sequence $\left\{B_{1}\right.$, $\left.B_{2}, \ldots, B_{k}\right\}$. Then, by definition, there is a set $\left\{i_{1}, i_{2}, \ldots, i_{k}\right\}$ with $1 \leq i_{1}<i_{2}<\cdots<i_{k} \leq m$ such that $B_{1}$ is the component of $T\left(S_{i_{k}}, \alpha_{i_{k}}\right) \cap T\left(S_{i_{1}}, \alpha_{i_{1}}\right)$ containing $x$, and such that $B_{j}$, $j=2,3, \ldots, k$ is the component of $T\left(S_{i_{j-1}}, \alpha_{i_{j-1}}\right) \cap T\left(S_{i_{j}}, \alpha_{i_{j}}\right)$ containing $s_{i_{j-1}} \circ s_{i_{2}} \circ s_{i_{1}}(x)$. If there is a $B_{j}$ is of the form $B_{\left(*, i_{j} ; *\right)}$, that is, a component which is not near to $\alpha_{i_{j}}$, then $B_{j}=B_{\left(i_{k}, i_{1} ; *\right)}$ for $j=1 ; B_{j}=B_{\left(i_{j-1}, i_{j} ; *\right)}$ for $j \neq 1$.

When $j<k$, we have that $s_{i_{j}} \circ s_{i_{j-1}} \circ \cdots \circ s_{i_{1}}(x) \in s_{i_{j}}\left(B_{j}\right) \in B_{j+1}$. Since $s_{i_{j}}\left(B_{\left(*, i_{j} ; *\right)}\right)$ does not meet any component of the form $B_{\left(*, i_{j} ; *\right)}$ but itself, $B_{j+1}=B_{\left(i_{j}, * ; *\right)}$. Because $B_{j+1}$ lies in $T\left(S_{i_{j+1}}, \alpha_{i_{j+1}}\right)$, we have $B_{j+1}=B_{\left(i_{j}, i_{j+1} ; *\right)}$. Similarly, we can prove that $B_{1}=B_{\left(i_{k}, i_{i} ; *\right)}$ if $B_{k}=B_{\left(i_{k-1}, i_{k} ; *\right)}$.

Notice that each $B_{j}$ is only one of two types: either $B_{j}=B_{\left(i_{j-1}, i_{j} ; *\right)}$ or $B_{\left(i_{j}, i_{j+1} ; *\right)}$. The above arguments have shown that if one component $B_{j}$ in an accompanying sequence is of the first type, the others are the same as it. Thus, we are done. 


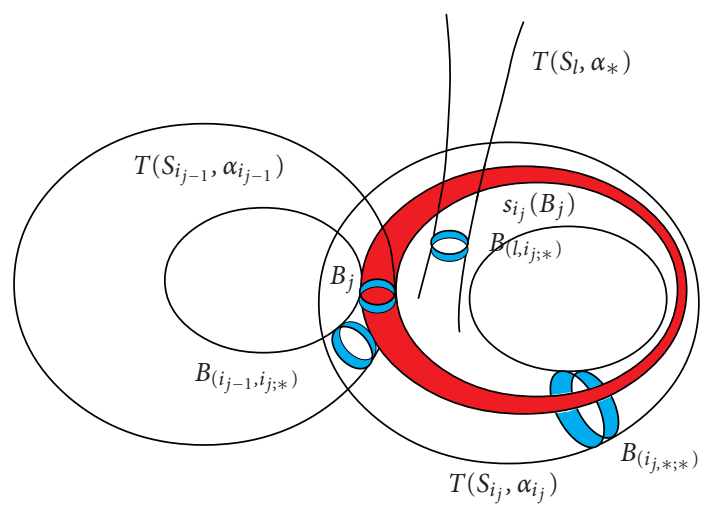

Figure 4.1

This lemma in fact implies that the components in one accompanying sequence are distinct. The next lemma will show that after some suitable isotopies on the slide homeomorphism, there is a one-to-one correspondence between the fixed point set on the sliding set and the set consisting of the above accompanying sequences.

Lemma 4.4. If the sliding set $\cup_{j=1}^{m} T\left(S_{j}, \alpha_{j}\right)$ is in general position, we can isotope the slide homeomorphisms $s\left(S_{i}, \alpha_{i}\right)$, relative to a neighborhood of $M-T\left(S_{i}, \alpha_{i}\right)$, to $s_{i}^{\prime}$, where $i=1$, $2, \ldots, m$, so that for each sequence $\mathscr{B}$ of components of the intersection of sliding sets of the form given in Lemma 4.3, there is unique fixed point of $f^{\prime}=s_{m}^{\prime} \circ s_{m-1}^{\prime} \circ \cdots \circ s_{1}^{\prime}$ with $\mathscr{B}$ as its accompanying sequence.

Moreover, the fixed point index of the fixed point of $f^{\prime}$ having

$$
\left\{B_{\left(i_{k}, i_{1} ; j_{1}\right)}, B_{\left(i_{1}, i_{2} ; j_{2}\right)}, B_{\left(i_{2}, i_{3} ; j_{3}\right)}, \ldots, B_{\left(i_{k-1}, i_{k} ; j_{k}\right)}\right\}
$$

as its accompanying sequence is $-I_{\left(i_{k}, i_{1} ; j_{1}\right)} I_{\left(i_{1}, i_{2} ; j_{2}\right)} \cdots I_{\left(i_{k-1}, i_{k} ; j_{k}\right)}$; the fixed point index of the fixed point of $f^{\prime}$ having

$$
\left\{B_{\left(i_{1}, i_{l} ; j_{1}\right)}, B_{\left(i_{2}, i_{1} ; j_{2}\right)}, B_{\left(i_{3}, i_{2} ; j_{3}\right)}, \ldots, B_{\left(i_{l}, i_{l-1} ; j_{l}\right)}\right\}
$$

as its accompanying sequence is $(-1)^{l} I_{\left(i_{1}, i_{l} ; j_{1}\right)} I_{\left(i_{2}, i_{1} ; j_{2}\right)} \cdots I_{\left(i_{l}, i_{l-1} ; j_{l}\right)}$, where $I_{(i, j ; k)}$ is the intersection number of the oriented path $\alpha_{i}$ and the oriented sphere $S_{j}$ in $M$ at the kth point $q_{(i, j ; k)}$ of $\alpha_{i} \cap S_{j}$.

Remark 4.5. Although $f^{\prime}=s_{m}^{\prime} \circ s_{m-1}^{\prime} \circ \cdots \circ s_{1}^{\prime}$ is no longer a composition of standard slide homeomorphisms $s_{j}^{\prime}$, we still say that $\left\{B_{1}, B_{2}, \ldots, B_{k}\right\}$ is the "accompanying sequence" of $x$ in the following sense: $B_{1}$ is the component of $T\left(S_{i_{k}}, \alpha_{i_{k}}\right) \cap T\left(S_{i_{1}}, \alpha_{i_{1}}\right)$ containing $x$, $B_{j}, j=2,3, \ldots, k$ is the component of $T\left(S_{i_{j-1}}, \alpha_{i_{j-1}}\right) \cap T\left(S_{i_{j}}, \alpha_{i_{j}}\right)$ containing $s_{i_{j-1}}^{\prime} \circ \cdots \circ s_{i_{2}}^{\prime} \circ$ $s_{i_{1}}^{\prime}(x)$. The proof of Lemma 4.1 still works as long as $s_{j}$ and $s_{j}^{\prime}$ have the same support set $T\left(S_{j}, \alpha_{j}\right)$ for each $j$.

Proof of Lemma 4.4. We will give the proof in three steps.

Step 1. Isotope each $s\left(S_{j}, \alpha_{j}\right)$. 
Consider an arbitrary component $B_{(i, j ; k)}$ of the intersection of the sliding sets. Since it is a component near the $k$ th point in $\alpha_{i} \cap S_{j}$, we can assume that

$$
\begin{aligned}
B_{(i, j ; k)} & \subset c_{i}^{-1}\left(\left\{(\theta, \varphi, t):\left|\theta-\hat{\theta}_{(i, j ; k ; i)}\right|<\delta, 0 \leq \varphi<2 \pi, 0<t<1\right\}\right) \\
& \subset c_{j}^{-1}\left(\left\{(\theta, \varphi, t):\left|\theta-\hat{\theta}_{(i, j ; k ; j)}\right|<\delta,\left|\varphi-\hat{\varphi}_{(i, j ; k ; j)}\right|<\delta, 0<t<1\right\}\right),
\end{aligned}
$$

where $\delta>0$, and all $\hat{\theta}_{(i, j ; k ; *)}$ and $\hat{\varphi}_{(i, j ; k ; *)}$ are constant. Note that the "length" of $B_{(i, j ; k)}$ in $T\left(S_{i}, \alpha_{i}\right)$ and the "area" of $B_{(i, j ; k)}$ in $T\left(S_{j}, \alpha_{j}\right)$ can be arbitrary small. The range of $c_{i}\left(B_{(i, j ; k)}\right)$ in $\theta$-coordinate, the range of $c_{j}\left(B_{(i, j ; k)}\right)$ in $\theta$-coordinate and the range of $c_{j}\left(B_{(i, j ; k)}\right)$ in $\varphi$ coordinate can be arbitrarily small. All of $B_{(*, * ; *)}$ 's share the same $\delta$.

By a small perturbation, we assume that the intervals $\left[\hat{\theta}_{(i, j ; k ; *)}-\delta, \hat{\theta}_{(i, j ; k ; *)}+\delta\right]$ and $\left[\hat{\varphi}_{(i, j ; k ; *)}-\delta, \hat{\varphi}_{(i, j ; k ; *)}+\delta\right]$ are disjoint for all possible $i, j, k, *$. Moreover, we can assume that

$$
\left[\hat{\theta}_{(i, j ; k ; i)}-\delta, \hat{\theta}_{(i, j ; k ; i)}+\delta\right] \subset\left(\pi, \frac{7 \pi}{6}\right), \quad\left[\hat{\theta}_{(i, j ; k ; j)}-\delta, \hat{\theta}_{(i, j ; k ; j)}+\delta\right] \subset\left(0, \frac{\pi}{6}\right) .
$$

For $i=1,2, \ldots, m$, we isotope $s\left(S_{i}, \alpha_{i}\right)$ to $s_{i}^{\prime}$ so that

$$
c_{i} s_{i}^{\prime} c_{i}^{-1}(\theta, \varphi, t)= \begin{cases}\left(2 \pi t+\frac{\pi}{6}, \varphi,-\frac{\theta}{2 \pi}+\frac{7}{12}\right) & \text { if } 0<\theta<\frac{\pi}{3}, \frac{5}{12}<t<\frac{7}{12}, \\ \left(2 \pi t-\frac{5 \pi}{6}, \varphi,-\frac{\theta}{2 \pi}+\frac{13}{12}\right) & \text { if } \pi<\theta<\frac{4 \pi}{3}, \frac{5}{12}<t<\frac{7}{12}, \\ (\theta+2 \pi t, \varphi, t) & \text { if } 0<t<\frac{1}{6} \text { or } \frac{5}{6}<t<1,\end{cases}
$$

and so that $s_{i}^{\prime}(x)=s_{i}(x)$ for $x \notin T\left(S_{i}, \alpha_{i}\right)$. Thus $s_{i}^{\prime}$ is isotopic to $s_{i}$ relative to $M-$ $c_{i}^{-1}(\{(\theta, \varphi, t): 1 / 6<t<5 / 6\})$. Thus, $f^{\prime}=s_{m}^{\prime} \circ s_{m-1}^{\prime} \circ \cdots \circ s_{1}^{\prime}$ is isotopic to $f$ relative to $M-\cup_{i=1}^{m} c_{i}^{-1}(\{(\theta, \varphi, t): 1 / 6<t<5 / 6\})$ which is a neighborhood of $M-\cup_{i=1}^{m} T\left(S_{i}, \alpha_{i}\right)$ in $M$.

Since $c_{j} \circ s_{j}^{\prime} c_{j}^{-1}$ preserves $\varphi$-levels, the condition that all possible intervals $\left[\hat{\varphi}_{(i, j ; k ; *)}-\right.$ $\left.\delta, \hat{\varphi}_{(i, j ; k ; *)}+\delta\right]$ are disjoint implies that $s_{j}^{\prime}\left(B_{(i, j ; k)}\right) \cap B_{\left(i^{\prime}, j ; k^{\prime}\right)}=\varnothing$ unless $i=i^{\prime}$ and $k=k^{\prime}$. Clearly, the sliding set here is in general position when $\delta$ is small enough. By Lemma 4.3, the accompanying sequence of each fixed point of $f^{\prime}$ is of one of two types listed there.

Step 2. Fixed points having accompanying sequences of the first type.

Consider a sequence $\left\{B_{\left(i_{k}, i_{1} ; j_{1}\right)}, B_{\left(i_{1}, i_{2} ; j_{2}\right)}, B_{\left(i_{2}, i_{3} ; j_{3}\right)}, \ldots, B_{\left(i_{k-1}, i_{k} ; j_{k}\right)}\right\}$ of the components of the intersection of sliding sets.

Since $B_{\left(i_{k}, i_{1} ; j_{1}\right)}$ ranges in $t$-direction from one component of $\partial T\left(S_{i_{1}}, \alpha_{i_{1}}\right)$ to the other component of $\partial T\left(S_{i_{1}}, \alpha_{i_{1}}\right)$, its image under $s_{i_{1}}^{\prime}$ will form a circle "parallel" to $\alpha_{i_{1}}$. Thus, $s_{i_{1}}^{\prime}\left(B_{\left(i_{k}, i_{1} ; j_{1}\right)}\right)$ meets any component of the form $B_{\left(i_{1}, * ; *\right)}$. Note that $B_{\left(i_{1}, i_{2} ; j_{2}\right)} \in c_{i_{1}}(\{(\theta, \varphi, t)$ : $\pi<\theta<7 \pi / 6\})$. The behavior of $s_{i_{1}}^{\prime}$ on $B_{\left(i_{k}, i_{1} ; j_{1}\right)}$ (see (4.6), (4.7), and (4.8)) implies that $s_{i_{1}}^{\prime}\left(B_{\left(i_{k}, i_{1} ; j_{1}\right)}\right)$ will be parallel to $B_{\left(i_{1}, i_{2} ; j_{2}\right)}$ in $\theta$-direction of $T\left(S_{i_{1}}, \alpha_{i_{1}}\right)$. Thus, $s_{i_{1}}^{\prime}\left(B_{\left(i_{k}, i_{1} ; j_{1}\right)}\right) \cap$ $B_{\left(i_{1}, i_{2} ; j_{2}\right)}$, which is a solid torus, also ranges in $t$-direction from one component of $\partial T\left(S_{i_{2}}\right.$, $\left.\alpha_{i_{2}}\right)$ to the other component of $\partial T\left(S_{i_{2}}, \alpha_{i_{2}}\right)$. Its image under $s_{i_{2}}$ meets $B_{\left(i_{2}, i_{3} ; j_{3}\right)}$. We then get a solid torus $s_{i_{2}}^{\prime}\left(s_{i_{1}}^{\prime}\left(B_{\left(i_{k}, i_{1} ; j_{1}\right)}\right) \cap B_{\left(i_{1}, i_{2} ; j_{2}\right)}\right) \cap B_{\left(i_{2}, i_{3} ; j_{3}\right)}=s_{i_{2}}^{\prime} \circ s_{i_{1}}^{\prime}\left(B_{\left(i_{k}, i_{1} ; j_{1}\right)}\right) \cap s_{i_{2}}^{\prime}\left(B_{\left(i_{1}, i_{2} ; j_{2}\right)}\right) \cap$ $B_{\left(i_{2}, i_{3} ; j_{3}\right)}$ in $B_{\left(i_{2}, i_{3} ; j_{3}\right)}$ (see Figure 4.2 ). 


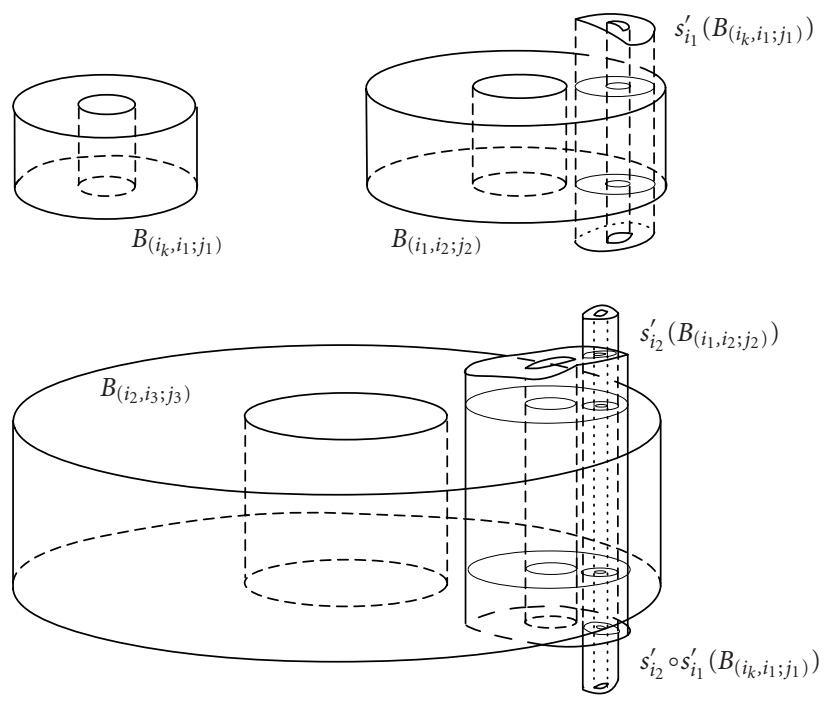

Figure 4.2

Repeating the above argument, we will get a solid torus in $B_{\left(i_{k}, i_{1} ; j_{1}\right)}$ :

$s_{i_{k}}^{\prime} \circ s_{i_{k-1}}^{\prime} \circ \cdots \circ s_{i_{1}}^{\prime}\left(B_{\left(i_{k}, i_{1} ; j_{1}\right)}\right) \cap s_{i_{k}}^{\prime} \circ s_{i_{k-1}}^{\prime} \circ \cdots \circ s_{i_{2}}^{\prime}\left(B_{\left(i_{1}, i_{2} ; j_{2}\right)}\right) \cap \cdots \cap s_{i_{k}}^{\prime}\left(B_{\left(i_{k-1}, i_{k} ; j_{k}\right)}\right) \cap B_{\left(i_{k}, i_{1} ; j_{1}\right)}$.

By Proposition 4.2 and the Remark 4.5 following the present lemma, a fixed point $x$ of $f^{\prime}$ will be contained in this set if $x$ has $\left\{B_{1}, B_{2}, \ldots, B_{k}\right\}$ as its accompanying sequence. Note that $f^{\prime}$ has unique fixed point on above set. Thus, the fixed point of $f^{\prime}$ with accompanying sequence $\left\{B_{1}, B_{2}, \ldots, B_{k}\right\}$ is unique.

Let $x_{*}$ be the unique fixed point of $f^{\prime}$ in the set in (4.9). Then $c_{i_{1}}\left(x_{*}\right)$ is a fixed point of $c_{i_{1}} \circ f^{\prime} \circ c_{i_{1}}^{-1}: U \rightarrow c_{i_{1}}\left(T\left(S_{i_{1}}, \alpha_{i_{1}}\right)\right)$, where $U$ is the $c_{i_{1}}$ image of the set in (4.9). Using the coordinates of $T^{2} \times I$, the three eigenvalues $\lambda_{1}, \lambda_{2}, \lambda_{3}$ of the derivative of $c_{i_{1}} \circ f^{\prime} \circ c_{i_{1}}^{-1}$ at $c_{i_{1}}\left(x_{*}\right)$ will satisfy the condition: one eigenvalue has absolute value greater than 1 , the other two have absolute values less than 1 . We assume that $\left|\lambda_{1}\right|>1,\left|\lambda_{2}\right|<1$ and $\left|\lambda_{3}\right|<1$.

From Figure 4.3, we know that the $\theta$-direction of $B_{(i, j ; k)}$ is mapped by $s_{j}^{\prime}$ into the $\theta$ direction of $B_{(j, * ; *)}$ if $I_{(i, j ; k)}>0$; the $\theta$-direction of $B_{\left(i, j ; k^{\prime}\right)}$ is mapped by $s_{j}^{\prime}$ into opposition of the $\theta$-direction of $B_{(j, * ; *)}$ if $I_{\left(i, j ; k^{\prime}\right)}<0$. Thus, the eigenvalue $\lambda_{1}>1$ if $I_{\left(i_{k}, i_{1} ; j_{1}\right)} I_{\left(i_{1}, i_{2} ; j_{2}\right)} \cdots$ $I_{\left(i_{k-1}, i_{k} ; j_{k}\right)}=1$; and $\lambda_{1}<-1$ if $I_{\left(i_{k}, i_{1} ; j_{1}\right)} I_{\left(i_{1}, i_{2} ; j_{2}\right)} \cdots I_{\left(i_{k-1}, i_{k} ; j_{k}\right)}=-1$.

Note that the fixed point index of an isolated fixed point of a map is just $(-1)^{\kappa}$, where $\kappa$ is the number of real eigenvalues which are greater than 1 of the derivative of this map at this fixed point, provided that 1 is not an eigenvalue of this derivative (see [4, page12, $3.2(2)])$. We have that ind $\left(c_{i_{1}} \circ f^{\prime} \circ c_{i_{1}}^{-1}, c_{i_{1}}\left(x_{*}\right)\right)=-I_{\left(i_{k}, i_{1} ; j_{1}\right)} I_{\left(i_{1}, i_{2} ; j_{2}\right)} \cdots I_{\left(i_{k-1}, i_{k} ; j_{k}\right)}$, which is also the fixed point index ind $\left(f^{\prime}, x_{*}\right)$ by the commutativity of fixed point index.

Step 3. Fixed points having accompanying sequences of the second type. 


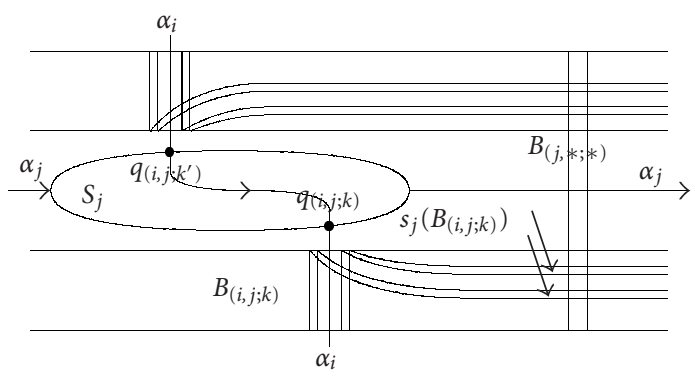

Figure 4.3

Note that the inverse $\left(f^{\prime}\right)^{-1}=\bar{s}_{1}^{\prime} \circ \bar{s}_{2}^{\prime} \circ \cdots \circ \bar{s}_{m}^{\prime}$ of $f^{\prime}$ is also a homeomorphism composed of finite isotoped slide homeomorphisms, where each $\bar{s}_{j}^{\prime}$ is isotopic to the slide homeomorphism $s\left(S_{j},-\alpha_{j}\right)$ determined by $S_{j}$ and the inverse $-\alpha_{j}$ of path $\alpha_{j}$. Clearly, the fixed point sets of $f^{\prime}$ and $\left(f^{\prime}\right)^{-1}$ are the same. Moreover, a fixed point of $f^{\prime}$ having $\left\{B_{\left(i_{1}, i_{l} ; *\right)}, B_{\left(i_{2}, i_{1} ; *\right)}, B_{\left(i_{3}, i_{2} ; *\right)}, \ldots, B_{\left(i_{1}, i_{l-1} ; *\right)}\right\}$ as its accompanying sequence is also a fixed point of $\left(f^{\prime}\right)^{-1}$ have an accompanying sequence of the first type discussed in last step.

Using the same argument as above, we can prove that there is a unique fixed point $y_{*}$ of $f^{\prime}$ having $\left\{B_{\left(i_{1}, i_{l} ; *\right)}, B_{\left(i_{2}, i_{1} ; *\right)}, B_{\left(i_{3}, i_{2} ; *\right)}, \ldots, B_{\left(i_{l}, i_{l-1} ; *\right)}\right\}$ as its accompanying sequence. The only difference is in the fixed point index. Because the three eigenvalues $\lambda_{1}, \lambda_{2}, \lambda_{3}$ of the derivative of $c_{i_{1}} \circ\left(f^{\prime}\right)^{-1} \circ c_{i_{1}}^{-1}$ at $c_{i_{1}}\left(y_{*}\right)$ satisfy the conditions: $\left|\lambda_{1}\right|>1,\left|\lambda_{2}\right|<1$ and $\left|\lambda_{3}\right|<1$, the three eigenvalues $\mu_{1}, \mu_{2}, \mu_{3}$ of the derivative of $c_{i_{1}} \circ f^{\prime} \circ c_{i_{1}}^{-1}$ at $c_{i_{1}}\left(y_{*}\right)$ will satisfy the conditions: $\left|\mu_{1}\right|=\left|1 / \lambda_{1}\right|<1,\left|\mu_{2}\right|=\left|1 / \lambda_{2}\right|>1$ and $\left|\mu_{3}\right|=\left|1 / \lambda_{3}\right|>1$. Since both of $f^{\prime}$ and $f$ are orientation-preserving, we have that $\lambda_{1} \lambda_{2} \lambda_{3}>0$ and $\mu_{1} \mu_{2} \mu_{3}>0$.

Note that at a point in $\alpha_{i} \cap S_{j}$, the algebraic intersection number of $\alpha_{i}$ with $S_{j}$ is opposite to the algebraic intersection number of $-\alpha_{i}$ with $S_{j}$. If $\left(-I_{\left(i_{1}, i_{l} ; j_{1}\right)}\right)\left(-I_{\left(i_{2}, i_{1} ; j_{2}\right)}\right) \cdots$ $\left(-I_{\left(i_{l}, i_{l-1} ; j_{l}\right)}\right)=(-1)^{l} I_{\left(i_{1}, i_{l} ; j_{1}\right)} I_{\left(i_{2}, i_{1} ; j_{2}\right)} \cdots I_{\left(i_{l}, i_{l-1} ; j_{l}\right)}>0$, by using the proof of the last step, we have that $\lambda_{1}>1$, and therefore $0<\mu_{1}=1 / \lambda_{1}<1$. Thus, $\mu_{2} \mu_{3}>0$. There are three possibilities: (1) $\mu_{2}, \mu_{3}>1$, (2) $\mu_{2}, \mu_{3}<-1$ and (3) $\mu_{2}$ and $\mu_{3}$ are conjugate complex numbers. In each case, the number of real eigenvalues which are greater than 1 is even. We have that $\operatorname{ind}\left(f^{\prime}, y_{*}\right)=\operatorname{ind}\left(c_{i_{1}} \circ f^{\prime} \circ c_{i_{1}}^{-1}, c_{i_{1}}\left(y_{*}\right)\right)=1$.

If $(-1)^{l} I_{\left(i_{1}, i_{l} ; j_{1}\right)} I_{\left(i_{2}, i_{1} ; j_{2}\right)} \cdots I_{\left(i_{l}, i_{l-1} ; j_{l}\right)}<0$, by using the proof of last step, we have that $\lambda_{1}<$ -1 , and therefore $-1<\mu_{1}=1 / \lambda_{1}<0$. Thus, $\mu_{2} \mu_{3}<0$. Hence, either $\mu_{2}<-1, \mu_{3}>1$ or $\mu_{2}>1, \mu_{3}<-1$. It follows that there is only one real eigenvalue which is greater than 1 , so $\operatorname{ind}\left(f^{\prime}, y_{*}\right)=\operatorname{ind}\left(c_{i_{1}} \circ f^{\prime} \circ c_{i_{1}}^{-1}, c_{i_{1}}\left(y_{*}\right)\right)=-1$.

Combining these two cases, we have that ind $\left(f^{\prime}, y_{*}\right)=(-1)^{l} I_{\left(i_{1}, i_{l} ; j_{1}\right)} I_{\left(i_{2}, i_{1} ; j_{2}\right)} \cdots I_{\left(i_{l}, i_{l-1} ; j_{l}\right)}$.

This lemma is a generalization of [9, Lemma 4.2]. The proof here is more descriptive than the direct computation there. The fixed point class coordinates of these fixed points can be computed in the same way.

Proposition 4.6. Let $f=s\left(S_{m}, \alpha_{m}\right) \circ s\left(S_{m-1}, \alpha_{m-1}\right) \circ \cdots \circ s\left(S_{1}, \alpha_{1}\right)$ be a homeomorphism composed of finitely many slide homeomorphisms. Assume that the $S_{j}$ 's are pairwise disjoint, 
and that any $\alpha_{i}$ and any $S_{j}, i, j=1,2, \ldots, m(i \neq j)$, intersect transversally. Then $f$ is isotopic to a homeomorphism with

$$
\begin{aligned}
\sum_{1 \leq j_{1}<\cdots<j_{k} \leq m}\left(\left|\alpha_{j_{k}} \cap S_{j_{1}}\right|\left|\alpha_{j_{1}} \cap S_{j_{2}}\right| \cdots\left|\alpha_{j_{k-1}} \cap S_{j_{k}}\right|\right. \\
\left.\quad+\left|\alpha_{j_{1}} \cap S_{j_{k}}\right|\left|\alpha_{j_{2}} \cap S_{j_{1}}\right| \cdots\left|\alpha_{j_{k}} \cap S_{j_{k-1}}\right|\right)
\end{aligned}
$$

fixed points.

Proof. The assumptions on $\alpha_{i}$ 's and $S_{k}$ 's imply that we can arrange the union of all sliding stes in general position provided $T\left(S_{i}, \alpha_{i}\right)$ is close to $\alpha_{i} \cup S_{i}$ for each $i$. Using above lemma and Lemma 3.4, we get immediately our conclusion.

By this proposition, the number $\left|\alpha_{i} \cap S_{j}\right|$ determines in some sense the number of fixed points. In order to reduce the number of fixed points of such homeomorphisms, the intersection numbers $\left(\left|\alpha_{i} \cap S_{j}\right|\right.$ 's) should be reduced. In [9, page 184], we defined

$$
M I\left(\alpha_{i}, S_{j}\right)=: \min \left\{\left|\alpha \cap S_{j}\right|: \alpha \simeq \alpha_{j} \operatorname{rel}\{0,1\}, \alpha \text { has no self intersection }\right\}
$$

From this definition, we have

Proposition 4.7. Let $S_{j}$ be an oriented sphere isotopic to a component of the boundary $\partial M_{k(j)}^{\prime}$ of a summand $M_{k(j)}^{\prime}$, and let $\left\langle\alpha_{i}\right\rangle=a_{1} b_{1} a_{2} b_{2} \cdots a_{n} b_{n} a_{n+1}$ where $b_{l}$ consists of words in $\pi_{1}\left(M_{k(j)}^{\prime}\right)$, $a_{l}$ does not contain any word in $\pi_{1}\left(M_{k(j)}^{\prime}\right)$ and all $a_{l}$ 's and $b_{l}$ 's are non-trivial except possibly for $a_{1}$ and $a_{n+1}$. Then $M I\left(\alpha_{i}, S_{j}\right)=2 n$ if $M_{k(j)}^{\prime} \neq S^{2} \times I ; M I\left(\alpha_{i}, S_{j}\right)=n$ if $M_{k(j)}^{\prime} \cong S^{2} \times I$. Here, the number $n$ is just the number of word "groups" of $\left\langle\alpha_{i}\right\rangle$, consisting of the words from $\pi_{1}\left(M_{k(j)}^{\prime}\right)$.

In particular, we have $\operatorname{MI}\left(\alpha_{i}, S_{j}\right)=0$ if $S_{j}$ is isotopic to $S_{i}$.

Proof. See [9, Proposition 4.4].

It should be noticed that all MI's can not be minimized at same time if any two $S_{i}$ 's are disjoint and if there are isotopic sliding spheres.

Example 4.8. Let $M=T_{1}^{3} \# T_{2}^{3} \# T_{3}^{3}$ be the connected sum of three 3-dimensional tori. For $j=1,2,3$, we write $g_{j 1}, g_{j 2}$ and $g_{j 3}$ for the generators of the free abelian group $\pi_{1}\left(T_{j}^{3}\right)$.

Let $S_{1}$ and $S_{3}$ be oriented spheres isotopic to the boundary of the summand $T_{1}^{3}-$ $\operatorname{Int}\left(D^{3}\right)$, and $S_{2}$ an oriented sphere isotopic to the boundary of the summand $T_{2}^{3}-$ $\operatorname{Int}\left(D^{3}\right)$. Three paths are given by $\left\langle\alpha_{1}\right\rangle=g_{21},\left\langle\alpha_{2}\right\rangle=g_{32} g_{12} g_{33}$, and $\left\langle\alpha_{3}\right\rangle=g_{31} g_{22} g_{33} g_{23}$ (see Figure 4.4).

The numbers of $\left|\alpha_{i} \cap S_{j}\right|$ 's in two cases are listed as follows:

\begin{tabular}{|c|c|c|c|}
\hline & $\alpha_{1}$ & $\alpha_{2}$ & $\alpha_{3}$ \\
\hline$S_{1}$ & - & 2 & 2 \\
\hline$S_{2}$ & 2 & - & 4 \\
\hline$S_{3}$ & 0 & 2 & - \\
\hline
\end{tabular}

\begin{tabular}{|c|c|c|c|}
\hline & $\alpha_{1}$ & $\alpha_{2}$ & $\alpha_{3}$ \\
\hline$S_{1}$ & - & 2 & 0 \\
\hline$S_{2}$ & 2 & - & 4 \\
\hline$S_{3}$ & 2 & 2 & - \\
\hline
\end{tabular}



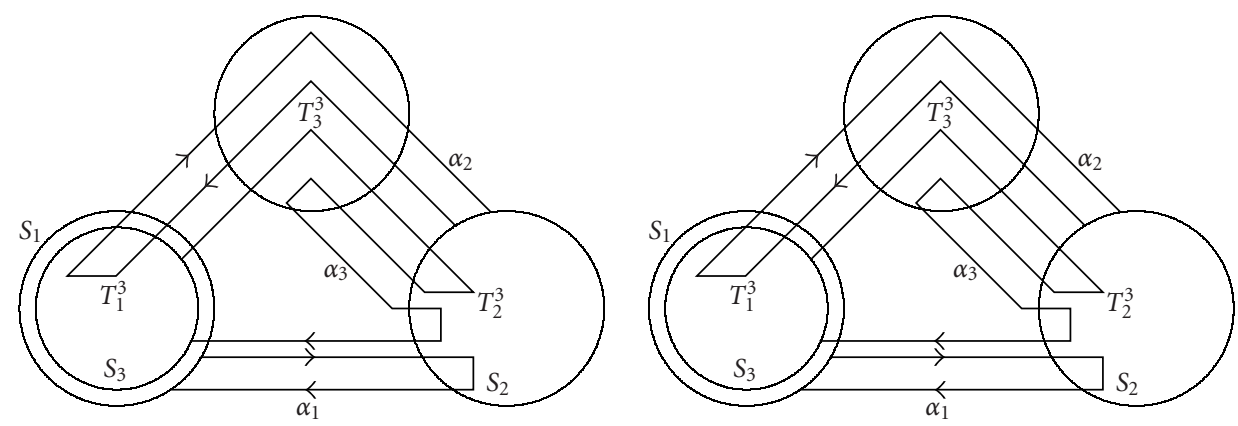

Figure 4.4

Thus, the sum in Proposition 4.6 is

$$
\begin{aligned}
\left(\left|\alpha_{3} \cap S_{1}\right|\left|\alpha_{1} \cap S_{2}\right|\left|\alpha_{2} \cap S_{3}\right|+\left|\alpha_{1} \cap S_{3}\right|\left|\alpha_{2} \cap S_{1}\right|\left|\alpha_{3} \cap S_{2}\right|\right) \\
\quad+\left(\left|\alpha_{2} \cap S_{1}\right|\left|\alpha_{1} \cap S_{2}\right|+\left|\alpha_{1} \cap S_{2}\right|\left|\alpha_{2} \cap S_{1}\right|\right) \\
\quad+\left(\left|\alpha_{3} \cap S_{1}\right|\left|\alpha_{1} \cap S_{3}\right|+\left|\alpha_{1} \cap S_{3}\right|\left|\alpha_{3} \cap S_{1}\right|\right) \\
\quad+\left(\left|\alpha_{3} \cap S_{2}\right|\left|\alpha_{2} \cap S_{3}\right|+\left|\alpha_{2} \cap S_{3}\right|\left|\alpha_{3} \cap S_{2}\right|\right)
\end{aligned}
$$

In the case shown on the left, it is $(8+0)+(4+4)+(0+0)+(8+8)=32$; in the other case, it is $(0+16)+(4+4)+(0+0)+(8+8)=40$.

Note that in both cases $\left|\alpha_{i} \cap S_{j}\right|=M I\left(\alpha_{i}, S_{j}\right)$ except for $(i, j)=(1,3)$ or $(3,1)$. Since $S_{3}$ and $S_{1}$ are isotopic, we have that $M I\left(\alpha_{1}, S_{3}\right)=M I\left(\alpha_{3}, S_{1}\right)=0$. But, these two numbers can not be realized simultaneously if the intersection of $S_{1}$ and $S_{3}$ is assumed to be empty.

Thus, we need the following.

Definition 4.9. Given slide homeomorphisms $s\left(S_{1}, \alpha_{1}\right), s\left(S_{1}, \alpha_{1}\right), \ldots, s\left(S_{m}, \alpha_{m}\right)$ whose composition is $f$, we define $M I\left(\left\{\alpha_{1}, \ldots, \alpha_{m}\right\},\left\{S_{1}, \ldots, S_{m}\right\}\right)$ to be:

$$
\begin{aligned}
\min _{\alpha_{j}^{\prime}, S_{j}^{\prime}} \sum_{1 \leq j_{1}<\cdots<j_{k} \leq m}\left(\left|\alpha_{j_{k}}^{\prime} \cap S_{j_{1}}^{\prime}\right|\left|\alpha_{j_{1}}^{\prime} \cap S_{j_{2}}^{\prime}\right| \cdots\left|\alpha_{j_{k-1}}^{\prime} \cap S_{j_{k}}^{\prime}\right|\right. \\
\left.+\left|\alpha_{j_{1}}^{\prime} \cap S_{j_{k}}^{\prime}\right|\left|\alpha_{j_{2}}^{\prime} \cap S_{j_{1}}^{\prime}\right| \cdots\left|\alpha_{j_{k}}^{\prime} \cap S_{j_{k-1}}^{\prime}\right|\right)
\end{aligned}
$$

where each $\alpha_{j}^{\prime}$ and $S_{j}^{\prime}, j=1,2, \ldots, m$, range over all oriented paths and spheres such that $\alpha_{j}^{\prime}$ and $S_{j}^{\prime}$ are isotopic to $\alpha_{j}$ and $S_{j}$, respectively, with $\alpha_{j}^{\prime} \cap S_{j}^{\prime}=\left\{\alpha_{j}^{\prime}(0), \alpha_{j}^{\prime}(1)\right\}$, and such that any two $\alpha_{i}^{\prime}$ 's and any two $S_{j}^{\prime}$ 's have empty intersection.

In Example 4.8, we have $M I\left(\left\{\alpha_{1}, \alpha_{2}, \alpha_{3}\right\},\left\{S_{1}, S_{2}, S_{3}\right\}\right)=32$. The relation between this "totally" minimal intersection number and the individual MI's is given by the following proposition. 
Proposition 4.10. The number $M I\left(\left\{\alpha_{1}, \ldots, \alpha_{m}\right\},\left\{S_{1}, \ldots, S_{m}\right\}\right)$ is greater or equal to the following sum:

$$
\begin{aligned}
\sum_{1 \leq j_{1}<\cdots<j_{k} \leq m}( & M I\left(\alpha_{j_{k}}, S_{j_{1}}\right) M I\left(\alpha_{j_{1}}, S_{j_{2}}\right) \cdots M I\left(\alpha_{j_{k-1}}, S_{j_{k}}\right) \\
& \left.+M I\left(\alpha_{j_{1}}, S_{j_{k}}\right) M I\left(\alpha_{j_{2}}, S_{j_{1}}\right) \cdots M I\left(\alpha_{j_{k}}, S_{j_{k-1}}\right)\right) .
\end{aligned}
$$

If any two sliding spheres are not isotopic, then the above two numbers are the same.

Now, we can state our main theorem.

THeOREM 4.11. Let $f=s\left(S_{m}, \alpha_{m}\right) \circ s\left(S_{m-1}, \alpha_{m-1}\right) \circ \cdots \circ s\left(S_{1}, \alpha_{1}\right)$ be a homeomorphism which is composed of finitely many slide homeomorphisms. Then, $f$ is isotopic to a homeomorphism with $\operatorname{MI}\left(\left\{\alpha_{1}, \ldots, \alpha_{m}\right\},\left\{S_{1}, \ldots, S_{m}\right\}\right)$ fixed points.

Proof. By definition, $\operatorname{MI}\left(\left\{\alpha_{1}, \ldots, \alpha_{m}\right\},\left\{S_{1}, \ldots, S_{m}\right\}\right)$ can be realized as

$$
\begin{aligned}
\sum_{1 \leq j_{1}<\cdots<j_{k} \leq m} & \left(\left|\alpha_{j_{k}}^{\prime} \cap S_{j_{1}}^{\prime}\right|\left|\alpha_{j_{1}}^{\prime} \cap S_{j_{2}}^{\prime}\right| \cdots\left|\alpha_{j_{k-1}}^{\prime} \cap S_{j_{k}}^{\prime}\right|\right. \\
& \left.+\left|\alpha_{j_{1}}^{\prime} \cap S_{j_{k}}^{\prime}\right|\left|\alpha_{j_{2}}^{\prime} \cap S_{j_{1}}^{\prime}\right| \cdots\left|\alpha_{j_{k}}^{\prime} \cap S_{j_{k-1}}^{\prime}\right|\right),
\end{aligned}
$$

where for each $j=1,2, \ldots, m, \alpha_{j}^{\prime}$ and $S_{j}^{\prime}$ are isotopic to $\alpha_{j}$ and $S_{j}$, respectively with $\alpha_{j}^{\prime} \cap$ $S_{j}^{\prime}=\left\{\alpha_{j}^{\prime}(0), \alpha_{j}^{\prime}(1)\right\}$, and that any two $\alpha_{i}^{\prime}$ 's and any two $S_{j}^{\prime}$ 's have no intersections. Thus, $s\left(S_{j}^{\prime}, \alpha_{j}^{\prime}\right)$ is isotopic to $s\left(S_{j}, \alpha_{j}\right)$. Applying Proposition 4.6 to the homeomorphism $s\left(S_{m}^{\prime}, \alpha_{m}^{\prime}\right) \circ s\left(S_{m-1}^{\prime}, \alpha_{m-1}^{\prime}\right) \circ \cdots \circ s\left(S_{1}^{\prime}, \alpha_{1}^{\prime}\right)$, we will obtain our conclusion.

By the lower bound property of Nielsen number, we immediately get the following corollary.

Corollary 4.12.

$$
0 \leq N(f) \leq M I\left(\left\{\alpha_{1}, \ldots, \alpha_{m}\right\},\left\{S_{1}, \ldots, S_{m}\right\}\right)
$$

\section{Some remarks}

In this final section, we will show that in some cases, the fixed point numbers can be further reduced.

Consider our homeomorphism $f$. If some successive sliding spheres, say $S_{n}, S_{n+1}, \ldots$, $S_{n+p}$, are isotopic, we have

$$
s\left(S_{n+p}, \alpha_{n+p}\right) \circ \cdots \circ s\left(S_{n+1}, \alpha_{n+1}\right) \circ s\left(S_{n}, \alpha_{n}\right)=s\left(S_{n}, \beta_{n}\right),
$$

where $\left\langle\beta_{n}\right\rangle=\left\langle\alpha_{n} \alpha_{n+1} \cdots \alpha_{n+p}\right\rangle$.

Combine all possible slide homeomorphisms which are in succession and have the same sliding spheres. We will get a shorter expression for $f$, denoted as follows:

$$
f=s\left(S_{m_{p}}, \beta_{m_{p}}\right) \circ \cdots \circ s\left(S_{m_{2}}, \beta_{m_{2}}\right) \circ s\left(S_{m_{1}}, \beta_{m_{1}}\right), \quad m_{p} \leq m .
$$


Using the main theorem (Theorem 4.11), we can isotope $f$ to a homeomorphism with

$$
M I\left(\left\{\beta_{m_{1}}, \beta_{m_{2}}, \ldots, \beta_{m_{p}}\right\},\left\{S_{m_{1}}, S_{m_{2}}, \ldots, S_{m_{p}}\right\}\right)
$$

fixed points. This number is no more than $\operatorname{MI}\left(\left\{\alpha_{1}, \ldots, \alpha_{m}\right\},\left\{S_{1}, \ldots, S_{m}\right\}\right)$.

In some cases, the two sliding spheres on two ends of the original expression of $f$ are isotopic, that is, $S_{m}$ is isotopic to $S_{1}$. This implies that $S_{m_{p}}$ is isotopic to $S_{m_{1}}$. Consider the homeomorphism

$$
\begin{aligned}
g & =s\left(S_{m_{1}}, \beta_{m_{1}}\right) \circ s\left(S_{m_{p}}, \beta_{m_{p}}\right) \circ s\left(S_{m_{p-1}}, \beta_{m_{p-1}}\right) \circ \cdots \circ s\left(S_{m_{2}}, \beta_{m_{2}}\right) \\
& =s\left(S_{m_{p}}, \beta_{m_{p}} \beta_{m_{1}}\right) \circ s\left(S_{m_{p-1}}, \beta_{m_{p-1}}\right) \circ \cdots \circ s\left(S_{m_{2}}, \beta_{m_{2}}\right) .
\end{aligned}
$$

Here, $\beta_{m_{p}} \beta_{m_{1}}$ can be considered as a path satisfying $\left\langle\beta_{m_{p}} \beta_{m_{1}}\right\rangle=\left\langle\beta_{m_{p}}\right\rangle\left\langle\beta_{m_{1}}\right\rangle$. Notice that $g=s\left(S_{m_{1}}, \beta_{m_{1}}\right) \circ f \circ\left(s\left(S_{m_{1}}, \beta_{m_{1}}\right)\right)^{-1}$, that is, $g$ is conjugate to $f$. The fixed point set of $f$ and $g$ are the same. Such a relation is preserved under isotopy. Thus, using the main theorem (Theorem 4.11) again, we can isotope $f$ to a homeomorphism with $\operatorname{MI}\left(\left\{\beta_{m_{2}}, \ldots\right.\right.$, $\left.\left.\beta_{m_{p-1}}, \beta_{m_{p}} \beta_{m_{1}}\right\},\left\{S_{m_{2}}, \ldots, S_{m_{p}}\right\}\right)$ fixed points.

Furthermore, if $\left\langle\beta_{m_{p}} \beta_{m_{1}}\right\rangle=1 \in \pi_{1}(M)$, we get that $g=s\left(S_{m_{p-1}}, \beta_{m_{p-1}}\right) \circ \cdots \circ s\left(S_{m_{2}}\right.$, $\left.\beta_{m_{2}}\right)$, and therefore the resulting fixed point number is just $M I\left(\left\{\beta_{m_{2}}, \ldots, \beta_{m_{p-1}},\right\},\left\{S_{m_{2}}, \ldots\right.\right.$, $\left.S_{m_{p-1}}\right\}$ ), so we can repeat the above procedure if $S_{m_{p-1}}$ is isotopic to $S_{m_{2}}$.

Apply this method to Example 4.8, we will get a new homeomorphism

$$
g=s\left(S_{3}, \alpha_{3} \alpha_{1}\right) \circ s\left(S_{2}, \alpha_{2}\right)=s\left(S_{3},\left\langle g_{31} g_{22} g_{33} g_{23} g_{21}\right\rangle\right) \circ s\left(S_{2},\left\langle g_{32} g_{12} g_{33}\right\rangle\right) .
$$

Thus, the homeomorphism is isotopic to one with $\operatorname{MI}\left(\left\{\alpha_{2}, \alpha_{3} \alpha_{1}\right\},\left\{S_{2}, S_{3}\right\}\right)=16$ fixed points, and so is the homeomorphism $s\left(S_{3}, \alpha_{3}\right) \circ s\left(S_{2}, \alpha_{2}\right) \circ s\left(S_{1}, \alpha_{1}\right)$.

\section{Acknowledgment}

This paper was supported by Natural Science Foundation of Beijing. I would like to thank the referee for many helpful comments and suggestions.

\section{References}

[1] R. F. Brown, The Lefschetz Fixed Point Theorem, Scott, Foresman, Illinois, 1971.

[2] D. Fried, Homological identities for closed orbits, Inventiones Mathematicae 71 (1983), no. 2, 419-442.

[3] R. W. Ghrist, P. J. Holmes, and M. C. Sullivan, Knots and Links in Three-Dimensional Flows, Lecture Notes in Mathematics, vol. 1654, Springer, Berlin, 1997.

[4] B. J. Jiang, Lectures on Nielsen Fixed Point Theory, Contemporary Mathematics, vol. 14, American Mathematical Society, Rhode Island, 1983.

[5] B. J. Jiang and J. H. Guo, Fixed points of surface diffeomorphisms, Pacific Journal of Mathematics 160 (1993), no. 1, 67-89.

[6] B. J. Jiang, S. Wang, and Y.-Q. Wu, Homeomorphisms of 3-manifolds and the realization of Nielsen number, Communications in Analysis and Geometry 9 (2001), no. 4, 825-877.

[7] M. R. Kelly, The Nielsen number as an isotopy invariant, Topology and its Applications 62 (1995), no. $2,127-143$. 
[8] D. McCullough, Mappings of reducible 3-manifolds, Geometric and Algebraic Topology, Banach Center Publ., vol. 18, PWN-Polish Scientific, Warsaw, 1986, pp. 61-76.

[9] X. Zhao, On the Nielsen numbers of slide homeomorphisms on 3-manifolds, Topology and its Applications 136 (2004), no. 1-3, 169-188.

Xuezhi Zhao: Department of Mathematics, Capital Normal University, Beijing 100037, China E-mail address: zhaoxve@mail.cnu.edu.cn 